\title{
Coherent dissipative soliton intermittency in ultrafast fiber lasers
}

\author{
Yueqing Du (杜岳卿)*, Chao Zeng (曾 超), Zhiwen He (贺志文), Qun Gao (高 群), and Dong Mao (毛 东) \\ Key Laboratory of Light Field Manipulation and Information Acquisition, Ministry of Industry and Information Technology, MOE Key Laboratory of Material \\ Physics and Chemistry under Extraordinary Conditions, Shaanxi Key Laboratory of Optical Information Technology, School of Physical Science and \\ Technology, Northwestern Polytechnical University, Xi'an 710072, China \\ *Corresponding author: yqdu@nwpu.edu.cn \\ Received May 17, 2021 | Accepted July 7, 2021 | Posted Online September 15, 2021
}

\begin{abstract}
As a universal phenomenon in nonlinear optical systems, intermittency is usually accompanied by the coherence loss such as soliton explosions in fiber lasers. Based on real-time spectroscopy, we revealed the coherent dissipative soliton intermittency in normal-dispersion fiber lasers. By increasing the pump strength, the intermittency transforms from the transient pulsation to the bi-stable soliton. It is demonstrated that the slow-gain effect dominates such coherent intermittency. Our results provide novel insights into laser physics, offering a promising approach for studying the bi-stable dissipative soliton.
\end{abstract}

Keywords: mode-locked lasers; optical solitons; fiber lasers.

DOI: 10.3788/COL202220.011401

\section{Introduction}

Ultrafast lasers play key roles in scientific fields such as optical clocks, optical precision measurement, and materials processing $^{[1]}$. Relying on the delicate interplay among gain, loss, dispersion, and nonlinearity, the ultrashort pulse, known as the dissipative soliton (DS) ${ }^{[2]}$, can maintain the stability in laser cavities. Instability of the DS arises from the nonlinearity in lasers, which can be described by the generalized nonlinear Schrodinger equation. Revealing physical mechanisms that drive the DS far from the stationary state is an ongoing challenge. Recently, thanks to widely applied real-time measurement techniques such as the dispersive Fourier transform (DFT) and time-lens ${ }^{[3,4]}$, diverse non-trivial transient phenomena have been observed in ultrafast lasers. The build-up of mode locking reveals the process wherein the initial noise self-localizes into a soliton in lasers ${ }^{[5-7]}$. The DS can evolve periodically with time via the Hopf bifurcation ${ }^{[8-10]}$. Dynamic competition between physical effects, e.g., gain/loss and spectral filtering by nonlinear spectral broadening, can induce diverse pulsating solitons ${ }^{[1-16]}$. Circulating in the laser cavity, the soliton emits resonant waves due to the periodic spatial modulation, generating fantastic pulsating behaviors ${ }^{[17,18]}$.

Apart from the widely researched pulsating solitons, the intermittency is rarely researched in ultrafast lasers, manifesting as the state where the optical wave keeps the metastable state for a long time while transiently entering the striking state, followed by the recovery of the metastable state ${ }^{[19]}$. A well-known intermittency is the soliton explosion ${ }^{[8,20-27]}$, in which the coherent DS transiently collapses into an incoherent exploding state ascribed to the nonlinear mixing with background noises. In contrast to the soliton explosion, the incoherent noise-like pulse can also recover to a coherent DS abruptly ${ }^{[28]}$. Besides, the intermittency can manifest as the transiently amplified dispersive waves ${ }^{[29]}$. Up to now, all the intermittencies in lasers are accompanied by the loss of coherence except for the collisioninduced soliton explosion ${ }^{[30]}$. A question of theoretical value is whether the DS during the intermittency can maintain its coherence without structure collapse. In the perspective of applications, the robust coherent intermittency, if being controlled, has potentials in chaos generation from lasers, random number generation, and high-energy femtosecond pulse for material processing.

In this Letter, the coherent DS intermittency, whose evolution strongly relies on the gain dynamics, is observed experimentally in the fiber laser. For lower pump power, the DS switches between the weak and extreme pulsations without loss of coherence. By increasing the pump power, the laser outputs the bi-stable DS, i.e., the DS that switches between two coherent states with different wavelengths and intensities. The laser is prone to annihilate into the random mode-locking state with high pump power. Experimental results demonstrate the dominance of slow-gain dynamics on the intermittency. Further mechanisms of the intermittency remain to be explored by the accurate numerical simulation that contains the slow dynamics of the gain medium. 


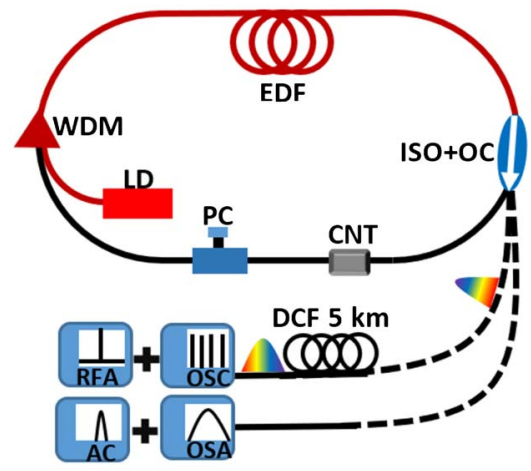

Fig. 1. Schematic of the fiber laser. EDF, Er-doped fiber; ISO, isolator; OC, output coupler; CNT, carbon nanotube; PC, polarization controller; LD, laser diode; WDM, wavelength division multiplexer; DCF, dispersion compensation fiber; OSC, oscilloscope; RFA, radio frequency analyzer; OSA, optical spectrum analyzer; $A C$, autocorrelator.

\section{Experimental Setup}

The schematic of the fiber laser is shown in Fig. 1. The cavity comprises an $8 \mathrm{~m}$ Er-doped fiber (EDF) and a $4 \mathrm{~m}$ single-mode fiber (SMF) with net dispersions of 21.5 and $-22 \mathrm{ps}^{2} / \mathrm{km}$, respectively, according to which the net dispersion is calculated to be $0.084 \mathrm{ps}^{2}$. The integrated module of the isolator and output coupler (10:90) ensures the unidirectional operation as well as extraction of the pulse train. Mode locking is realized by the carbon-nanotube (CNT) film. The cavity is pumped by a $975 \mathrm{~nm}$ laser diode through the 980/1550 wavelength division multiplexer. The polarization controller (PC) is tuned to control the mode-locking state. A real-time oscilloscope together with the $5 \mathrm{~km}$ dispersion compensation fiber $(750 \mathrm{ps} / \mathrm{nm})$ implements the real-time spectral measurement based on the DFT technique. Despite all of the components in this cavity being polarization-insensitive, the PC can still manipulate the lasing state via three effects: (i) polarization burn hole in the gain medium, (ii) nonlinear polarization coupling between two orthogonal components, and (iii) lumped loss due to the squeezing on the fiber.

\section{Experimental Results}

Tuning the pump power slightly above the threshold of mode locking, the intermittent state is obtained as shown in Fig. 2. The burrs on the time-averaged spectrum [measured by an optical spectrum analyzer (OSA)] indicate special transient events during the DS evolution [see Fig. 2(a)]. The average width of the DS is 7.13 ps according to the autocorrelation trace in Fig. 2(b), wherein the smooth profile validates the coherence of the mode-locked pulse that is distinct from the noise-like pulse. The non-stationary mode-locking state is confirmed via modulating the sidebands on the radio frequency (RF) spectrum in Fig. 2(c). The normalized peak intensity of the time-stretch signals versus roundtrips (RTs) is shown in Fig. 2(d). Different from the conventional pulsating DS with stable


Fig. 2. Mode-locking state at the pump power of $25.35 \mathrm{~mW}$. (a) Spectrum measured by OSA, (b) autocorrelation trace, (c) RF spectrum, and (d) peak intensity evolution of the normalized DFT signals over 85,000 RTs.

oscillating amplitude and period ${ }^{[18]}$, here, the evolution of the spectral intensity over 85,000 RTs is composed of the extreme and weak pulsations without mode-locking annihilation, which can be regarded as the intermittency rather than the regular pulsation [Fig. 2(d)]. The broadband spectrum, smooth profile of the autocorrelation trace, and clear peaks on the RF spectrum fully demonstrate that the lasing state is a coherent mode-locked state with time-varying properties.

To reveal the details of intermittency, real-time spectral evolution is plotted in Fig. 3. As shown in Figs. 3(a) and 3(b), the weak pulsation transforms into a transition state with slight fluctuations, followed by the extreme pulsation with strong spectral peaks induced by the self-phase-modulation (SPM) [Figs. 3(a) and $3(\mathrm{~b})$ ]. The periods of the weak and extreme pulsations are 327 and 338 RTs, corresponding to frequencies of 52.7 and $51 \mathrm{kHz}$, respectively, in agreement with the measured value

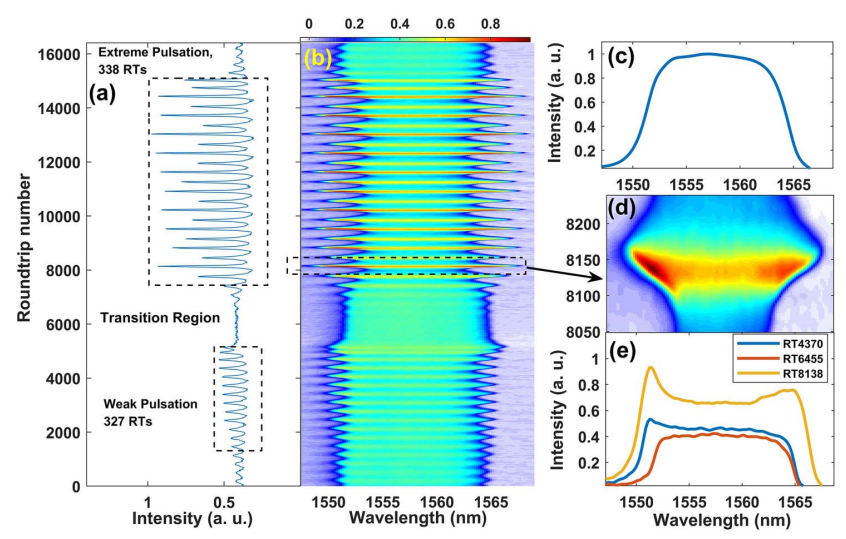

Fig. 3. Shot-to-shot evolution of the pulse at the pump power of $25.35 \mathrm{~mW}$. (a) Intensity evolution of normalized DFT signals over 16,000 RTs, (b) spatialspectral evolution, (c) averaged spectrum measured by DFT, (d) local enlargement of the marked area, and (e) single-shot spectra at three RTs. 
of $49 \mathrm{kHz}$ from the RF spectrum [Fig. 2(c)]. Despite the exotic evolution of the DS, the average spectrum over 16,000 RTs in Fig. 3(c) agrees qualitatively with that measured by OSA. The SPM-induced transient spectral broadening during the extreme pulsation [see Fig. 3(d)] accounts for the burrs of the spectrum measured by the OSA [see Fig. 2(a)]. One can note that two basic stages, i.e., weak and extreme pulsations, emerge alternatively, which is distinct from the pulsating DS. What is more, the coherent state is maintained during the switching between basic stages. In this regard, the unique phenomenon is named the coherent DS intermittency.

The dependence of the intermittency on the pump power is displayed in Fig. 4. By fixing the PC while gradually increasing the pump power, the intermittent pulsation gradually disappears [see Figs. 4(a)-4(c)]. The DS reaches the quasi-bi-stable state at the pump power of $28.2 \mathrm{~mW}$, switching between the high and low levels without pulsation [Figs. 4(d)-4(f)]. The duration of the low-level state for bi-stable DS decreases from 5241 to 2524 RTs as the pump power increases from 28.20 to $31.95 \mathrm{~mW}$. Further increasing the pump power to $32.85 \mathrm{~mW}$, the bi-stable DS annihilates into a stochastic state of the random mode-locking.

Detailed properties of the bi-stable DS at the pump power of $31.95 \mathrm{~mW}$, including average and real-time properties, are illustrated in Fig. 5. The OSA measured spectrum has steep edges with burrs induced by intermittency [Fig. 5(a)]. The sidebands on the RF spectrum indicate the $1.14 \mathrm{kHz}$ modulating frequency of the intermittency [Fig. 5(b)]. To reveal the wavelength shifting of the spectrum, the weighted central wavelength (WCW) of each single-shot spectrum is calculated by

$$
W=\int \lambda|S(\lambda)|^{2} \mathrm{~d} \lambda / \int|S(\lambda)|^{2} \mathrm{~d} \lambda,
$$

where $|S(\lambda)|^{2}$ and $\lambda$ are the power spectral density and vacuum wavelength, respectively. The WCW switches in sync with the spectral intensity [Fig. 5(c)], abruptly jumping with a step of

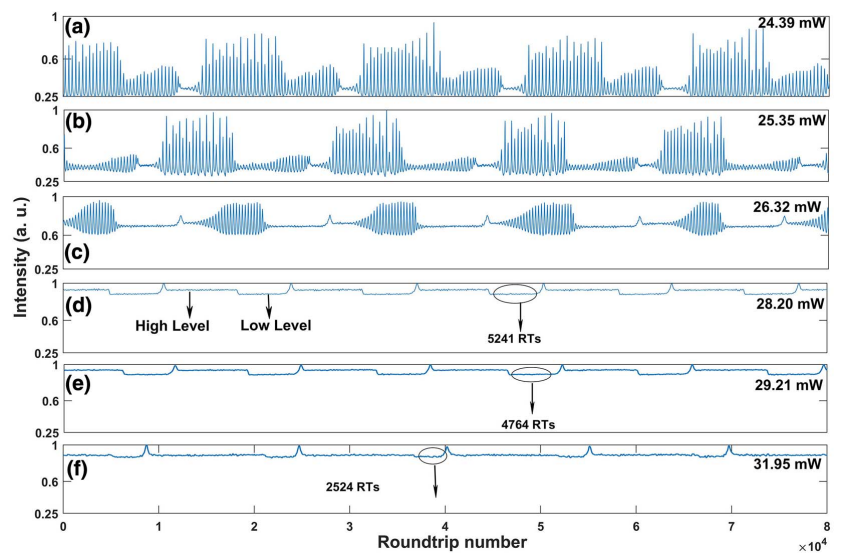

Fig. 4. Spectral intensity evolution of the normalized DFT signals over 80,000 RTs at different pump powers: (a) $24.39 \mathrm{~mW}$, (b) $25.35 \mathrm{~mW}$, (c) $26.32 \mathrm{~mW}$, (d) $28.20 \mathrm{~mW}$, (e) $29.21 \mathrm{~mW}$, and (f) $31.95 \mathrm{~mW}$.
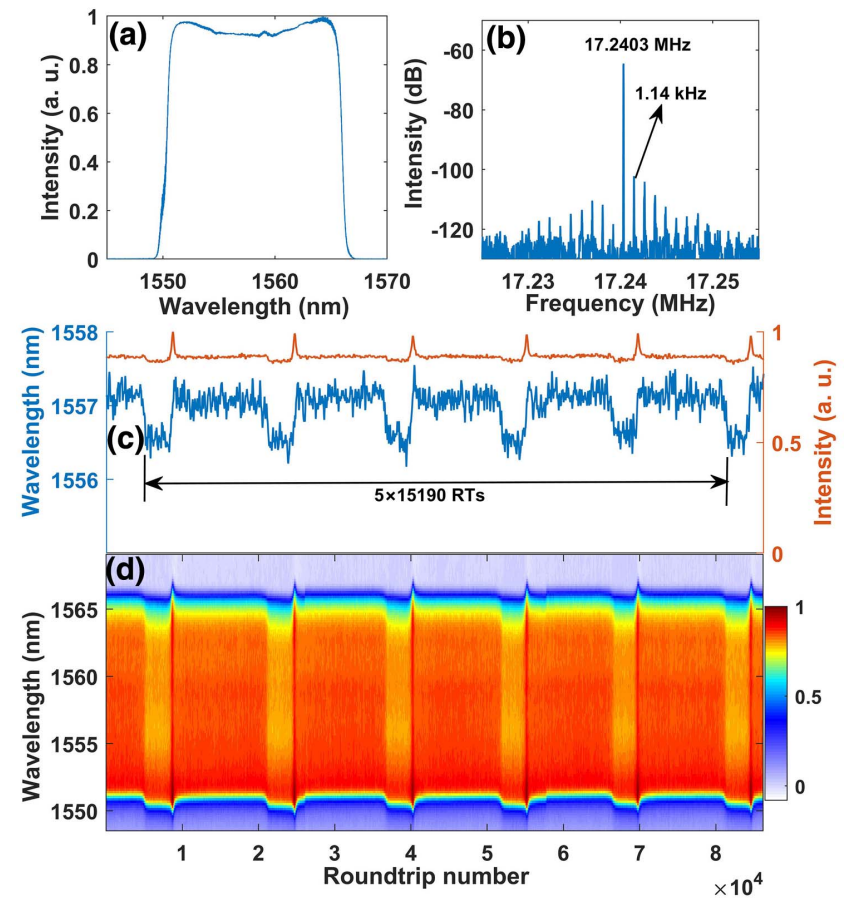

Fig. 5. Pulse state at the pump power of $31.95 \mathrm{~mW}$. (a) Optical spectrum measured by OSA, (b) RF spectrum, (c) center wavelength and intensity evolution, and (d) shot-to-shot evolution.

$\sim 0.6 \mathrm{~nm}$. The red and blue shifts arise in the switching toward the high and low levels, respectively. The average period of the bi-stable DS is $\sim 15,316 \mathrm{RTs}$, which agrees with the $1.14 \mathrm{kHz}$ modulation frequency of the RF spectrum. As shown in Fig. 5(d), the bi-stable DS in the form of intermittent wavelength switching is visualized by the spatial-spectral evolution.

In a previous work ${ }^{[10]}$, a kind of bi-stable soliton that switches between distinct states is observed in the laser mode-locked by the nonlinear polarization rotation, wherein the birefringenceinduced dual-pump filter promotes the bi-stability. Although similar to the case in Ref. [10], the DS in our work is modelocked by the polarization-insensitive CNT, excluding the possibility of filtering-induced wavelength switching. In experiments, we note a universal synchronization between longer (shorter) WCW and higher (lower) spectral intensity levels in the evolution of the bi-stable DS. The interaction between broadband DS and population inversion of the $\mathrm{Er}^{3+}$ ions can reshape the effective gain spectrum in the $\mathrm{EDF}^{[31,32]}$, triggering the switching of the DS between two states.

The random mode-locking state at the pump power of $32.85 \mathrm{~mW}$ is displayed in Fig. 6. Comparing the results with the those of intermittency, we summarize three similarities between them: (i) there are two peaks in the spectrum corresponding to the random mode locking at wavelengths of 1558.2 and $1558.99 \mathrm{~nm}$ [Fig. 6(a)], whose separation of $0.79 \mathrm{~nm}$ is similar to the wavelength jumping step of the bistable DS ( $0.6 \mathrm{~nm})$ (Fig. 5); (ii) the time intervals between adjacent random mode-locking events, 4874 and 8259 RTs, as 

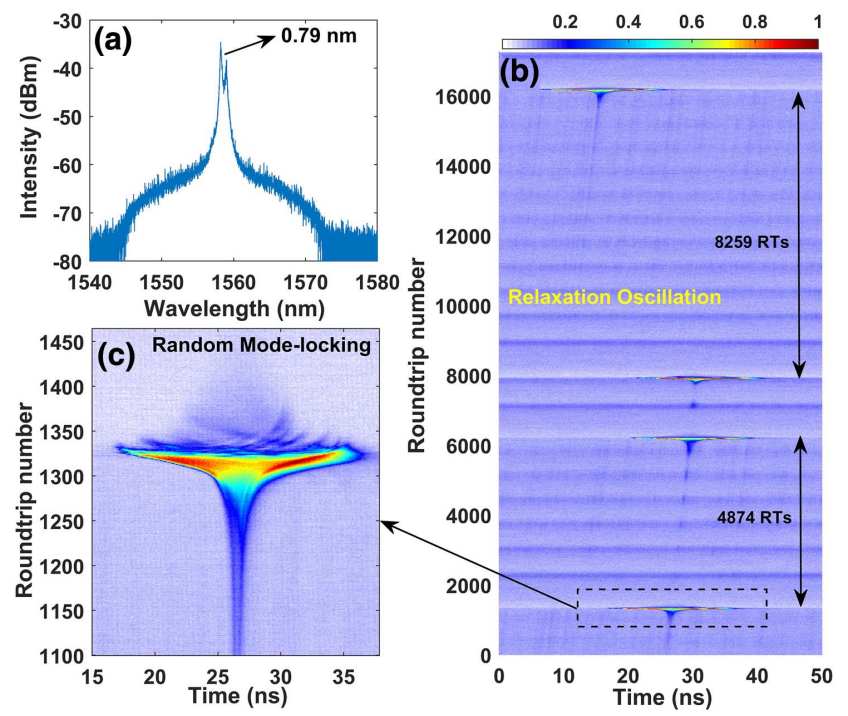

Fig. 6. Random mode locking at the pump power of $32.85 \mathrm{~mW}$. (a) Optical spectrum measured by OSA, (b) shot-to-shot evolution of the time-stretch signals, and (c) enlargement of the marked areas in (b).

marked in Fig. 6(b), have a similar magnitude to those of the bistable DS [Fig. 4(d) and 4(e)]; (iii) the periods of the relaxation oscillation in the random mode-locking [Fig. 6(b)] range from 698 to 741 RTs, which are the same order of magnitude as those in the intermittent pulsating DS [Fig. 3(a)].

A clear fact is that the slow-gain dynamics, i.e., the recovery and depletion of the population inversion of the $\mathrm{Er}^{3+}$ ions, determining the time-varying gain and the effective gain spectrum, dominates wave dynamics such as the temporal oscillation and wavelength shifting. Considering three similarities between the random mode locking and coherent DS intermittency, it is reasonable to speculate that the observed intermittency in Figs. 2-5 arises from the slow-gain dynamics. In addition, the pulse experiences an exotic spectral broadening during the random mode locking [Fig. 6(c)], in which the broadband state sustains for dozens of RTs, manifesting as the burring pedestal in the OSA measured spectrum [Fig. 6(a)]. After the random mode locking at high pump power emerges, the robust coherent intermittency can be recovered by decreasing the pump power, demonstrating that the CNT is not damaged at high pump power.

\section{Discussion}

In our work, despite the distinct evolutionary behaviors at different pump powers, the coherent DS intermittency is always composed of two basic stages, e.g., either weak and extreme pulsations at lower pump power or two quasi-stationary DSs with different wavelengths and intensities at higher pump power. It is not easy to understand why the basic stage of the intermittency transforms from the pulsating DS to the quasi-stationary DS with increasing pump power, however, the results of the pulsating DS in Ref. [12] give us enlightening thoughts. Peng et al. found that the pulsating DS emerges at lower pump power, while the stationary DS favors higher pump power, and such an experimental phenomenon is reproduced by numerical simulations with the average-field equation, wherein the pulsating DS is found to be related to the quintic terms of the gain. In this regard, we think the distinct basic stages of the intermittency at different pump powers have a similar origination to that in Ref. [12].

Spontaneous switching between basic stages is an important difference between DS intermittency and conventional pulsating $\mathrm{DS}^{[11-16]}$. The switching is a threshold behavior depending on the slow-gain dynamics, in which the population inversion, small-signal gain, as well as effective gain spectrum vary with time. When the time-varying gain reaches the threshold of switching, the pulse in the cavity is driven to transform from its current stage to another one. The above explanation needs to be confirmed by numerical simulations, which can be implemented by a complete simulation model that contains the slow-gain dynamics. Furthermore, one can note the extreme enhancement of the DS in the extreme pulsating stage [see Figs. 4(a)-4(c)] and the random mode locking (see Fig. 6), indicating the possibility of the slow-gain dynamics on triggering the rogue wave generation in ultrafast fiber lasers.

\section{Conclusion}

In conclusion, the coherent DS intermittency is experimentally revealed in the net-normal-dispersion fiber laser. Different from conventional pulsating solitons and chaotic solitons, the intermittency manifests as robust switching between basic stages, maintaining coherence during evolution. At the low pump power, the intermittency manifests periodic switching between different pulsating solitons. The laser generates bi-stable DS with periodic wavelength switching by further increasing the pump power. The dominance of the slow-gain dynamics is indicated by the similarities between the random mode locking and the coherent DS intermittency. Future work will focus on finding a convincing explanation through numerical simulations, which needs to be implemented through a complete model containing the interaction between the broadband DS and gain medium. Our work offers novel insights into the nonlinear transient dynamics of the DS, promoting the understanding of ultrafast intermittency in fiber lasers.

\section{Acknowledgement}

This work was supported by the National Natural Science Foundation of China (Nos. 61805277, 11634010, and 11874300), Natural Science Basic Research Program of Shaanxi (No. 2019JQ-447), Fundamental Research Funds for the Central Universities (No. 310202011QD003), National Key R\&D Program of China (No. 2017YFA0303800), and Fundamental Research Funds for the Central Universities (Nos. 3102019JC008 and 3102019PY002). 


\section{References}

1. H. L. Chen, X. T. Jiang, S. X. Xu, and H. Zhang, "Recent progress in multiwavelength fiber lasers: principles, status, and challenges," Chin. Opt. Lett. 18, 041405 (2020).

2. P. Grelu and N. Akhmediev, "Dissipative solitons for mode-locked lasers," Nat. Photon. 6, 84 (2012).

3. A. Mahjoubfar, D. V. Churkin, S. Barland, N. Broderick, S. K. Turitsyn, and B. Jalali, "Time stretch and its applications," Nat. Photon. 11, 341 (2017).

4. P. Ryczkowski, M. Närhi, C. Billet, J. M. Merolla, G. Genty, and J. M. Dudley, "Real-time full-field characterization of transient dissipative soliton dynamics in a mode-locked laser," Nat. Photon. 12, 221 (2018).

5. G. Herink, B. Jalali, C. Ropers, and D. R. Solli, "Resolving the build-up of femtosecond mode-locking with single-shot spectroscopy at $90 \mathrm{MHz}$ frame rate," Nat. Photon. 10, 321 (2016).

6. X. M. Liu, X.-K. Yao, and Y.-D. Cui, "Real-time observation of the buildup of soliton molecules," Phys. Rev. Lett. 121, 023905 (2018).

7. J. S. Peng and H. P. Zeng, "Build-up of dissipative optical soliton molecules via diverse soliton interactions," Laser Photon. Rev. 12, 1800009 (2018).

8. J. M. Soto-Crespo, N. Akhmediev, and A. Ankiewicz, "Pulsating, creeping, and erupting solitons in dissipative systems," Phys. Rev. Lett. 85, 2937 (2000).

9. J. M. Soto-Crespo, M. Grapinet, P. Grelu, and N. Akhmediev, "Bifurcations and multiple-period soliton pulsations in a passively mode-locked fiber laser," Phys. Rev. E 70, 066612 (2004).

10. C. Y. Bao, W. K. Chang, C. X. Yang, N. Akhmediev, and S. T. Cundiff, "Observation of coexisting dissipative solitons in a mode-locked fiber laser," Phys. Rev. Lett. 115, 253903 (2015).

11. Y. Q. Du, Z.-W. Xu, and X.-W. Shu, "Spatio-spectral dynamics of the pulsating dissipative solitons in a normal-dispersion fiber laser," Opt. Lett. 43, 3602 (2018).

12. J. S. Peng, S. Boscolo, Z. Zhao, and H. P. Zeng, "Breathing dissipative solitons in mode-locked fiber lasers," Sci. Adv. 5, eaax1110 (2019).

13. C. Chao, Q. Q. Huang, M. Al Araimi, A. Rozhin, S. Sergeyev, and C. B. Mou, "Observation of chaotic polarization attractors from a graphene mode locked soliton fiber laser," Chin. Opt. Lett. 17, 020012 (2019).

14. Z. Q. Wang, Q. Jiang, Z. Tang, and Z. Zhang, "Complex pulsating dynamics of counter-propagating solitons in a bidirectional ultrafast fiber laser," Opt. Express 28, 28209 (2020).

15. J. Chen, X. Zhao, T. Li, J. J. Yang, J. S. Liu, and Z. Zheng, "Generation and observation of ultrafast spectro-temporal dynamics of different pulsating solitons from a fiber laser," Opt. Express 28, 14127 (2020).
16. T. H. Xian, L. Zhan, W. C. Wang, and W. Y. Zhang, "Subharmonic entrainment breather solitons in ultrafast lasers," Phys. Rev. Lett. 125, 163901 (2020).

17. Y. Q. Du, M. M. Han, P. Y. Cheng, and X. W. Shu, "Pulsating soliton with broadened Kelly sidebands in an ultrafast fiber laser," Opt. Lett. 44, 4087 (2019).

18. M. Liu, Z. W. Wei, H. Li, T. J. Li, A. P. Luo, W. C. Xu, and Z. C. Luo, "Visualizing the "invisible" soliton pulsation in an ultrafast laser," Laser Photon. Rev. 14, 1900317 (2020).

19. S. H. Stogatz, Nonlinear Dynamicssa and Chaos (Perseus Books, 1994).

20. S. T. Cundiff, J. M. Soto-Crespo, and N. Akhmediev, "Experimental evidence for soliton explosions," Phys. Rev. Lett. 88, 073903 (2002).

21. O. Descalzi, C. Cartes, J. Cisternas, and H. R. Brand, "Exploding dissipative solitons: the analog of the Ruelle-Takens route for spatially localized solutions," Phys. Rev. E 83, 056214 (2011).

22. S. C. V. Latas and M. F. S. Ferreira, "Why can soliton explosions be controlled by higher-order effects?” Opt. Lett. 36, 3085 (2011).

23. S. C. V. Latas and M. F. S. Ferreira, "Soliton explosion control by higherorder effects," Opt. Lett. 35, 1771 (2010).

24. A. F. J. Runge, N. G. R. Broderick, and M. Erkintalo, "Observation of soliton explosions in a passively mode-locked fiber laser," Optica 2, 36 (2015).

25. M. Liu, A. P. Luo, W. C. Xu, and Z. C. Luo, "Dissipative rogue waves induced by soliton explosions in an ultrafast fiber laser," Opt. Lett. 41, 3912 (2016).

26. Y. Q. Du, M.-M. Han, and X.-W. Shu, "Dark solitons in the exploding pulsation of the bright dissipative soliton in ultrafast fiber lasers," Opt. Lett. 45, $666(2020)$

27. F. C. Meng, C. Lapre, C. Billet, G. Genty, and J. M. Dudley, "Instabilities in a dissipative soliton-similariton laser using a scalar iterative map," Opt. Lett. 45, $1232(2020)$.

28. Y. Q. Du, S. V. Sergeyev, Z. W. Xu, M. M. Han, X. W. Shu, and S. K. Turitsyn, "Alternation of the mode synchronization and desynchronization in ultrafast fiber laser," Laser Photon. Rev. 14, 1900219 (2020).

29. S. Lee, K. Park, H. Kim, L. A. Vazquez-Zuniga, J. Kim, and Y. Jeong, "Intermittent burst of a super rogue wave in the breathing multi-soliton regime of an anomalous fiber ring cavity," Opt. Express 26, 11447 (2018).

30. M. Liu, T.-J. Li, A.-P. Luo, W.-C. Xu, and Z.-C. Luo, “"Periodic" soliton explosions in a dual-wavelength mode-locked $\mathrm{Yb}$-doped fiber laser," Photon. Res. 8, 246 (2020).

31. C. R. Gile and S. E. Desurvire, "Modeling erbium-doped fiber amplifier," IEEE J. Lightwavave Technol. 9, 271 (1991).

32. P. Sidorenko, W. Fu, and F. W. Wise, "Nonlinear ultrafast fiber amplifiers beyond the gain-narrowing limit," Optica 6, 1328 (2019). 